\title{
Revisiting Newton's rings with a plasmonic optical flat for high-accuracy surface inspection
}

\author{
Yun Zheng ${ }^{1, *}$, Jie Bian ${ }^{1,}$, Xiao-Long Wang ${ }^{2}$, Ju-Xiu Liu ${ }^{1}$, Peng Feng ${ }^{1}$, Hai-Xiong Ge ${ }^{1}$, Olivier JF Martin ${ }^{2}$ \\ and Wei-Hua Zhang ${ }^{1}$
}

Two parallel optical surfaces often exhibit colorful fringes along the lines of equal thickness because of the interference of light. This simple phenomenon allows one to observe subwavelength corrugations on a reflective surface by simply placing on it a flat reference dielectric surface, a so-called optical flat, and inspecting the resultant interference pattern. In this work, we extend this principle from dielectric surfaces to two-dimensional plasmonic nanostructures. Optical couplings between an Au nanodisk array and an Au thin film were measured quantitatively using two different techniques, namely, the classical Newton's rings method and a closed-loop nano-positioning system. Extremely high spectral sensitivity to the inter-surface distance was observed in the near-field coupling regime, where a $1-\mathrm{nm}$ change in distance could alter the resonance wavelength by almost $10 \mathrm{~nm}$, $>40$ times greater than the variation in the case without near-field coupling. With the help of a numerical fitting technique, the resonance wavelength could be determined with a precision of $0.03 \mathrm{~nm}$, corresponding to a distance precision as high as $0.003 \mathrm{~nm}$. Utilizing this effect, we demonstrated that a plasmonic nanodisk array can be utilized as a plasmonic optical flat, with which nanometer-deep grooves can be directly visualized using a low-cost microscope.

Light: Science \& Applications (2016) 5, e16156; doi:10.1038/Isa.2016.156; published online 7 October 2016

Keywords: near-field optical coupling; optical flat; plasmonics

\section{INTRODUCTION}

The topographical information of a structured surface can be visualized as optical patterns via interference by simply placing that surface on a flat interface, namely, an optical flat ${ }^{1,2}$. As Isaac Newton established the relation between the interference pattern and the size of the gap between the two surfaces (that is, the local height information $)^{3}$, this interference-based technique has become one of the most widely used techniques for surface flatness inspection ${ }^{1,4}$. This simple technique allows one to estimate surface corrugations with an accuracy of $\sim 1 / 10$ of a wavelength, using the naked eye. However, if the surface corrugations are much smaller than the wavelength, then the interference patterns become undistinguishable because the contrast is determined by the ratio between the corrugation height and the wavelength. In physics, the insensitivity of light to deepsubwavelength objects shares the same root as the well-known diffraction limit, that is, the wave nature of light.

A straightforward means of improving the resolution is to increase the spatial frequency of the light (that is, to shorten the effective wavelength) by confining the light to the nanoscale 5 . Over the past few decades, a variety of techniques based on this concept have been developed, including near-field optical microscopy ${ }^{5-7}$, superlensing ${ }^{8}$ and plasmonics ${ }^{9}$. In particular, it has been demonstrated that the extreme light localization can be achieved with plasmonic nanostructures via their resonance modes, that is, the collective oscillations of the free electrons in metals ${ }^{10}$. The strong spatial confinement makes a plasmonic nanostructure highly sensitive to the environmental changes ${ }^{11,12}$, and even a minute change in the refractive index in the vicinity of such a nanostructure can lead to a considerable resonance shift. In particular, when another metal nanoparticle is present in close proximity, their resonance modes will hybridize and exhibit marked resonance shifts ${ }^{13,14}$, thus making plasmonic nanostructures an excellent tool for distance sensing. On the basis of this principle, plasmonic nanorulers have recently been demonstrated in various configurations, such as nanoparticle pairs ${ }^{15-17}$, nanorod pairs ${ }^{18,19}$, three-dimensional nanorulers ${ }^{20}$, plasmonic polarization spectro-tomography ${ }^{21}$ and particle-film coupled structures $^{22-24} 25,26$, to name a few.

Inspired by those works, we extend the concept of the classical optical flat from the world of dielectrics to the world of plasmonics and investigate the coupling behaviors between two dimensional (2D) nanostructured plasmonic surfaces. Indeed, interference phenomena between unconventional optical surfaces, so-called metasurfaces, made of dispersive materials have recently attracted considerable research interest because of their extraordinary optical properties. One prominent example is an ultrathin absorber based on the far-field interference of metasurface ${ }^{27-29}$. In this work, we focus on the optical

\footnotetext{
${ }^{1}$ College of Engineering and Applied Sciences, National Laboratory of Solid State Microstructures and Collaborative Innovation Center of Advanced Microstructures, Nanjing University, Nanjing 210093, China and ${ }^{2}$ Nanophotonics and Metrology Laboratory, Swiss Federal Institute of Technology Lausanne (EPFL), Lausanne 1015, Switzerland *These authors contributed equally to this work.

Correspondence: WH Zhang, Email: zwh@nju.edu.cn

Received 9 December 2015; revised 12 April 2016; accepted 13 April 2016; accepted article preview online 15 April 2016
} 
coupling mechanisms for 2D nanostructured plasmonic metasurfaces in the near-field regime, with the aim of developing a 'plasmonic optical flat' for fast, low-cost and high-accuracy surface inspection.

\section{MATERIALS AND METHODS}

\section{Fabrication of the plasmonic optical flat}

Our plasmonic optical flat consists of a 2D array of metal nanodisks with a subwavelength pitch size on a flat and transparent dielectric surface. Electron-beam lithography was used to fabricate samples with small dimensions $(60 \times 60 \mu \mathrm{m})$. Figure $1 \mathrm{~b}$ shows a scanning electron microscope (SEM) image of such a sample, which is composed of an array of $40-\mathrm{nm}$ thick, $100-\mathrm{nm}$ wide squared-shaped Au nanodisks with a $250-\mathrm{nm}$ pitch on a flat quartz substrate.

In addition to electron-beam lithography, nanoimprint lithography was used for the fabrication of large-format plasmonic optical flats, which were used for the high-accuracy surface inspection experiment reported in the later part of this work. In detail, a quartz plate was first patterned via nanoimprint lithography using a hole array template (200-nm pitch, 60-nm hole diameter), which was fabricated via interference lithography using a 266-nm laser (Coherent Inc., Santa Clara, CA, USA). Then, $40 \mathrm{~nm}$ of Au was deposited from the normal direction using an electron-beam evaporator. Finally, the resist was lifted off, and a plasmonic optical flat (that is, an Au nanodisk array) was obtained on the centimeter scale.

\section{Ultrafine distance tuning between two surfaces}

To study the quantitative dependence of the coupling on the distance in the near-field regime, we applied the Newton's rings method by placing a spherical glass surface coated with a $40-\mathrm{nm}$ Au film on the plasmonic optical flat to form a continuously varying air gap, as illustrated in Figure 1a. The gap size, $g$, satisfies the simple relation $g=g_{0}+R-\sqrt{R^{2}-r^{2}}$, where $R$ denotes the sphere radius, $g_{0}$ is the gap size at the center point and $r$ is the distance between the observation point and the center point. In the vicinity of the center point, $\mathrm{d} g / \mathrm{d} r$ is near zero, enabling ultrafine tuning of $g$ for highprecision measurements.

The success of such measurements relies on the accuracy and stability of the gap size $g_{0}$. To ensure stable and accurate control of $g_{0}$, we used quartz tuning fork sensors to monitor the forces between the two plasmonic surfaces and to keep the forces ${ }^{30}$, and consequently the gap size, constant $\left(g_{0}=5.4 \mathrm{~nm}\right)$ during the measurements with the assistance of a closed-loop control system ${ }^{30,31}$. This method allowed us to monitor and control $g_{0}$, with subnanometer precision. The detailed experimental data can be found in Supplementary Section 2.

It is worth mentioning that in principle, $g_{0}$ can be chosen freely, and here, we set it to $\sim 5 \mathrm{~nm}$ based on the considerations of coupling strength, resonance wavelength and mechanical forces. This distance is sufficiently small for studying near-field couplings, although the resonance remains within the spectral range of our spectrometer. If $g_{0}$ were smaller, the resonance wavelength would be $>1 \mu \mathrm{m}$, beyond the spectral range of our detector (Supplementary Section 1). In addition, a smaller $g_{0}$ would also increase the risk of the sample damage because of the stronger interaction forces.

\section{Optical characterization}

In the measurements, the spherical surface was gently positioned above the plasmonic disk array using a piezo stage. The coupled system was illuminated with white light from below, and the interference pattern formed in the air gap was collected from below using a $40 \times$ objective (numerical aperture $=0.9$ ). Finally, the reflection spectra along the radial direction of the Newton's rings were recorded using an imaging spectrometer equipped with a chargecoupled device (iDus 402, Andor, Belfast, Northern Ireland) ${ }^{32}$.

\section{Numerical simulations}

A commercial finite-difference time-domain (FDTD) solver (Lumerical Inc., Vancouver, BC, Canada) was used to simulate the near-field couplings between the $2 \mathrm{D}$ plasmonic nanostructures. In the model, periodic boundary conditions were used in the lateral directions, and a a

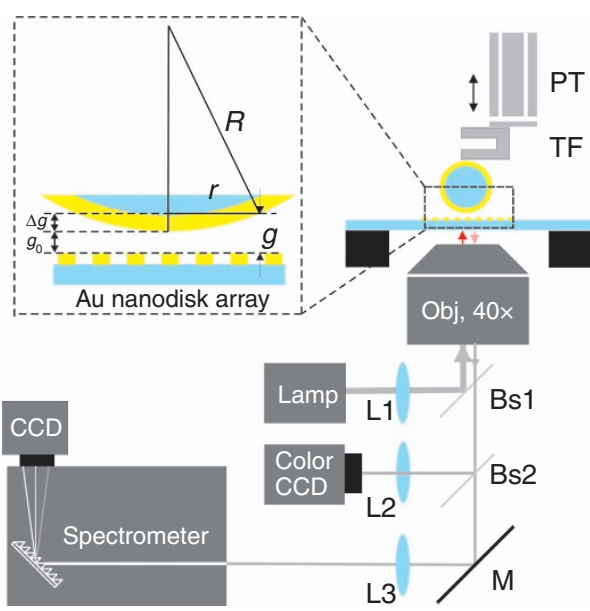

b

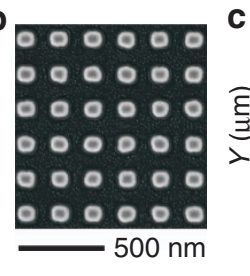

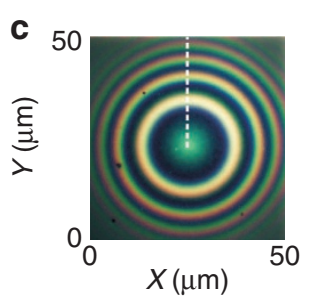

d

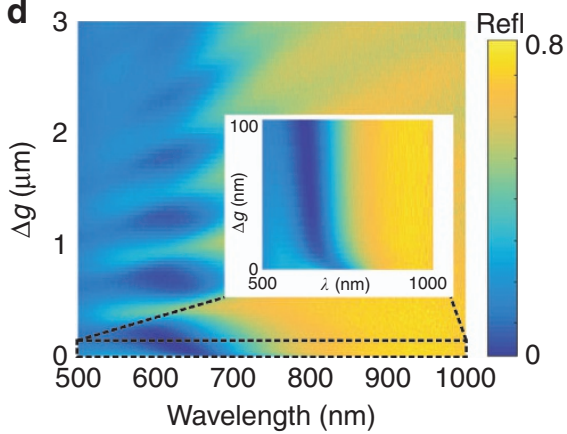

Figure 1 Plasmonic Newton's rings experiment. (a) Schematic drawing of the experimental setup. (b) SEM image of a typical plasmonic optical flat consisting of a 2D plasmonic nanodisk array on a quartz substrate. (c) Plasmonic Newton's rings captured by a color camera. (d) Reflection spectra measured along the dashed line in c. The inset shows the detailed spectral behaviors of the two plasmonic metasurfaces in the near-field coupling regime. In a, PT and TF denote the piezo tube and tuning fork, respectively. 
perfectly matched layer was used in the normal direction to avoid undesired reflections. The mesh size was $1 \times 1 \times 1 \mathrm{~nm}$. We simulated the reflection spectra at different gap sizes, determined the resonance wavelengths using those simulated spectra and finally calculated the charge distributions at the resonance wavelengths, using the divergence of the electric fields ${ }^{33}$.

\section{RESULTS AND DISCUSSION}

Figure 1c shows a typical plasmonic Newton's rings pattern generated between the plasmonic interfaces. It consists of a series of concentric rings with rainbow colors, similar to those formed between glass surfaces. However, these plasmonic Newton's rings exhibit certain significant differences from the conventional ones. A major difference is evident in the center area of the rings, particularly the near-touching area. In conventional Newton's rings, the center area is dark and colorless, and the pattern slowly brightens in the radial direction. By contrast, the center area of the plasmonic Newton's rings is bright and shows rich color changes.

\section{Large redshift induced by near-field coupling}

To reveal the spectral responses in detail, we measured the reflection spectra along the radial direction of the rings (dashed lines in Figure 1c). As shown in Figure 1d, there are a series of absorption peaks due to the near-field coupling of the two plasmonic surfaces. In the far-field regime, the wavelengths of the absorption peaks increase linearly with the gap size, as in the case of conventional Fabry-Pérot cavities. This is consistent with the results reported previously by other groups $^{34}$. However, in the near-touching regime, the resonance absorption peak behaves very differently. It shows marked redshifts when the gap size decreases (inset of Figure 1d), similar to the case of the coupled system of a single nanoparticle and an Au thin film predicted by one of the authors ${ }^{35}$ and observed by Smith and colleagues $^{23,36}$.

To study the spectral behaviors in the near-field coupling regime, we retrieved the resonance wavelength $\lambda_{\text {res }}$ by fitting it with a Lorentzian function and then plotted it as a function of the gap, $g$, as shown in Figure 2a. The resonance wavelength increases monotonically as $g$ decreases. When $g$ is very small, the resonance wavelength becomes extremely sensitive to the gap size, shifting by $33 \mathrm{~nm}$ when $g$ changes from 10 to $5 \mathrm{~nm}$. This high sensitivity makes such a plasmonic nanodisk array an excellent tool for high-resolution distance sensing. To describe the sensitivity of the system, we define the distance sensitivity, $s=\left|\mathrm{d} \lambda_{\text {res }} / \mathrm{d} g\right|$, that is, the slope of the $\lambda_{\text {res }}-g$ curve in Figure 2a, and plot $s$ as a function of $g$. As shown in
Figure 2b, $s=0.23$ when $g>30 \mathrm{~nm}$. However, when $g$ becomes $<30 \mathrm{~nm}$, $s$ increases rapidly and reaches 9.5 at $g=5 \mathrm{~nm}$, which is 41 times the value observed when $g>30 \mathrm{~nm}$.

\section{Resonance shift measured by mechanically tuning the nanogap} To validate the $\lambda_{\text {res }}-g$ relation in the near-field regime, a second method was developed to measure the reflection spectra at different gap sizes. Here, $g_{0}$ was varied continuously by ramping the driving voltage of the piezo stage, although reflection spectra were collected at a fixed position on the sample. We plotted the results together with those obtained using the Newton's rings technique, and the two curves were found to match each other perfectly, as shown in Figure 2a. This finding confirms the observed high distance sensitivity of the nearfield plasmonic coupling system.

\section{Gap-size sensing with picometer precision}

The resolution of this plasmonic near-field coupling-based distancesensing technique is determined by how precisely the resonance wavelength can be resolved. Because of the high material losses, the plasmon resonances are broad, with a full-width at half-maximum (FWHM) of $\sim 70 \mathrm{~nm}$. Numerical fitting is therefore necessary to precisely determine the resonance wavelength. In brief, we assume that in the vicinity of the resonance peaks, the absorption spectra can be described by a Lorentzian function; then, the spectra can be fitted using the least-squares method, which allows the uncertainty of the resonance wavelength to be calculated directly ${ }^{5,37}$. In our case, the resonance wavelength uncertainty $\Delta \lambda_{\text {res }}$ reached $0.03 \mathrm{~nm}$, three orders of magnitude smaller than the FWHM of the peak, similar to the super-resolution achieved in photo activated localization microscopy and stochastic optical reconstruction microscopy ${ }^{38,39}$. Therefore, in the near-field coupling regime, the distance between two plasmonic surfaces can be determined with an accuracy as high as $\Delta g=\Delta \lambda_{\text {res }} / s=0.003 \mathrm{~nm}$. This method yields measurements of changes or variations in gap size, not absolute distance measurements. Therefore, the roughness of the plasmonic nanodisk array does not influence the measurement accuracy.

Notably, the $0.003 \mathrm{~nm}$ precision reported here was obtained using incoherent white light. This is fundamentally different from conventional interference-based distance measurement techniques, in which monochromatic laser sources are always required to reach a high precision because the precision of the measurement is determined by the phase uncertainty of the light. Recently, a weak measurementbased technique has been implemented to improve the precision of measurements using white light sources; however, the reported
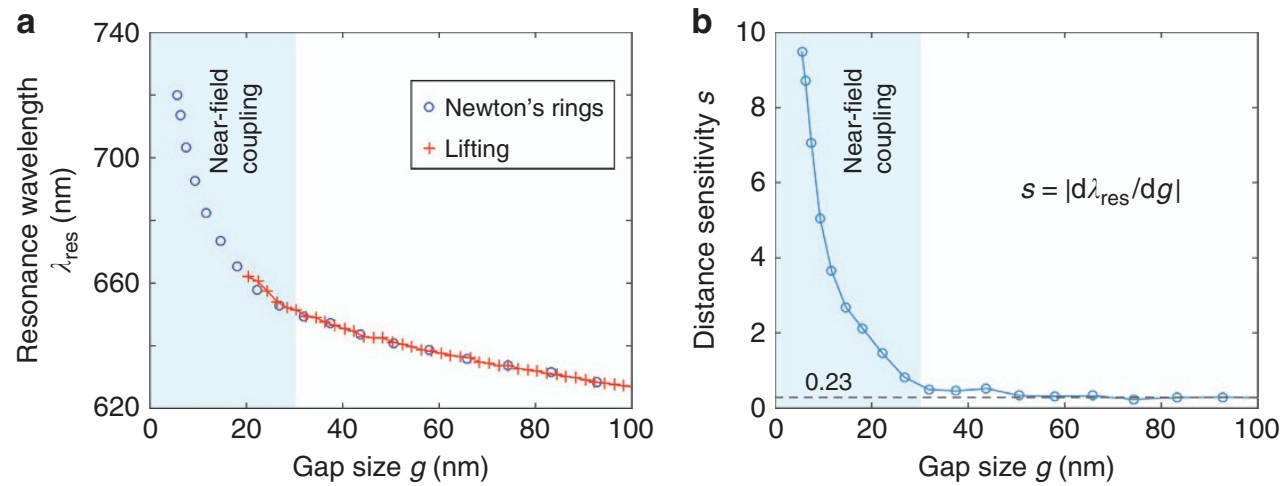

Figure 2 Resonance behavior of the coupled plasmonic metasurfaces. (a) Resonance wavelength, $\lambda_{\text {res, }}$ as a function of gap size, $g$, as measured using the Newton's rings method and the lifting method, in which the gap size was tuned using a piezo tube. (b) Distance sensitivity as a function of $g$ retrieved from the experimental data presented in a. The distance sensitivity, s, increases markedly when entering the near-field coupling regime. 
a

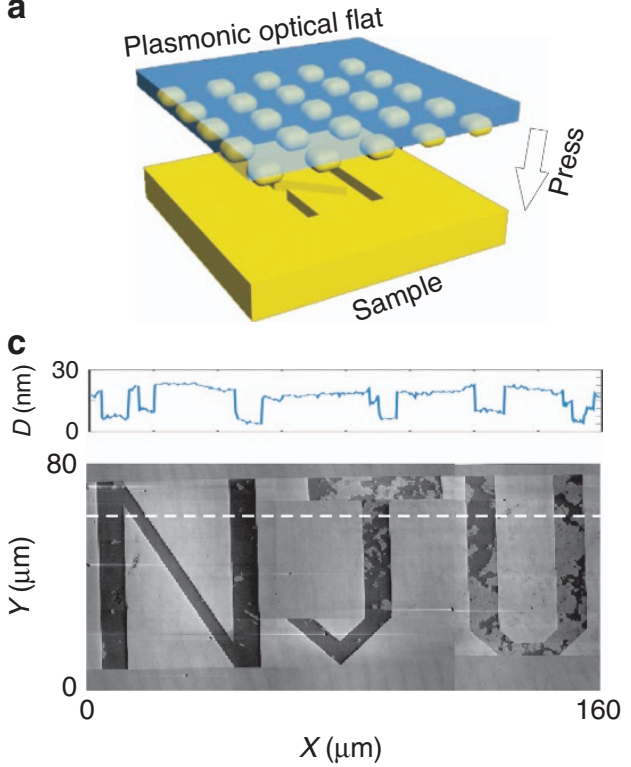

b

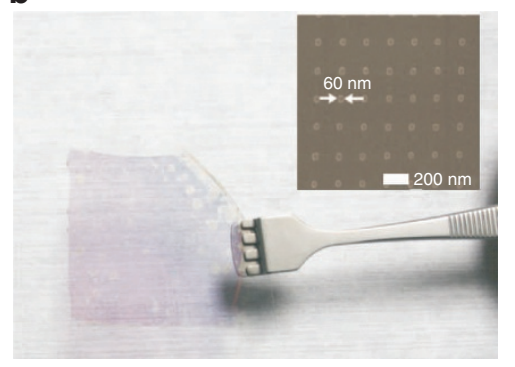

d

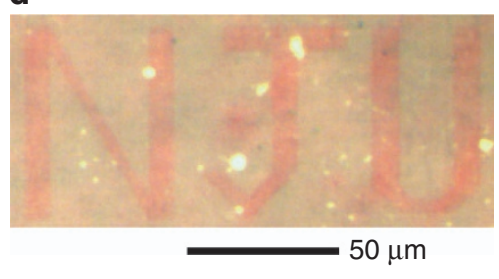

Figure 3 Optical profiling using a plasmonic optical flat. (a) Schematic drawing of the experiment. (b) Plasmonic optical flat fabricated using a nanoimprint-based method. The inset shows an SEM image of the 2D plasmonic nanodisk array. (c) AFM image of the 15-nm deep NJU-shaped grooves and their cross section along the dashed line. (d) Optical image of the nanogrooved film pressed onto the plasmonic optical flat. AFM, atomic force microscope.

equivalent distance uncertainty was $0.1 \mathrm{~nm}^{40}$, still two orders of magnitude short of our results.

Visualizing nanometer corrugations using the plasmonic optical flat The high distance sensitivity causes the 2D plasmonic nanodisk array to act as a high-performance plasmonic optical flat, which allows fast and high-accuracy inspection of the flatness of metal surfaces. To demonstrate this application, we fabricated a 15-nm deep NJU-shaped pattern on a polydimethylsiloxane substrate by duplicating the pattern from a Si template that was patterned using electron-beam lithography, as shown in Figure 3c. The plasmonic nanodisk array for generating the interference pattern was fabricated over a large area ( $\sim 1$ inch) using the nanoimprint technique, consisting of a $2 \mathrm{D}$ array of $60-\mathrm{nm}$ diameter Au nanodisks with a pitch size of $200 \mathrm{~nm}$; see Figure $3 \mathrm{~b}$. For the measurement, the patterned Au thin film was simply pressed onto the plasmonic optical flat, as illustrated in Figure $3 \mathrm{a}$, and the interference pattern was measured using a lowcost USB microscope. The 15-nm deep pattern was clearly visualized with good contrast, as shown in Figure $3 \mathrm{~d}$. In the groove area, the reflection is reddish, whereas in the remaining area where the two surfaces were in contact, the reflection is greenish. We also repeated the experiment using a conventional optical flat (that is, a glass substrate) instead of the plasmonic optical flat, and no pattern could be observed in that case.

Compared with the conventional optical flat, this plasmonic nearfield coupling-based profiling technique offers much higher distance sensitivity and allows the visualization of nanometer-scale corrugations on metal surfaces. Meanwhile, the new method inherits the merits of measurements using conventional optical flats, which are simple, fast and potentially low in cost. Especially now, with recent advances in nanofabrication techniques, for example, the nanoimprint technique, it has become possible to produce plasmonic nanostructure arrays over a large area at a low price ${ }^{41,42}$.
Strong near-field coupling revealed through numerical simulation The high distance sensitivity demonstrated above originates from the near-field couplings of the $2 \mathrm{D}$ plasmonic nanodisk array with the metal film. When one nanostructured plasmonic surface is placed close to another, mode hybridizations will occur because of the interactions of free charges in the metals ${ }^{13}$. Because these hybrid modes are much more localized than the normal cavity modes, the relative influence exerted on them by a change in distance is much stronger than that in the conventional case. This makes the coupled plasmonic system a perfect tool for distance sensing.

To elaborate on the above statement, we simulated the mode profiles of the resonances of a $2 \mathrm{D}$ plasmonic nanodisk array with and without near-field coupling, using the FDTD method. As illustrated in Figure $4 \mathrm{a}$ and $4 \mathrm{~d}$, at the resonance frequency, a unit nanodisk of the plasmonic nanodisk array behaves like a dipole, with charges distributed evenly on both the upper and lower surfaces of the nanodisk. When an additional metal surface is present in the near-field zone, mode hybridizations occur and charges become concentrated in the gap area because of the strong near-field coupling between free charges in the metal, as shown in Figure 4e. This enables high distance sensitivity in the coupled plasmonic system. In addition, the strong near-field coupling also leads to a very high electric field enhancement. In the case of a 10-nm gap size, the local field intensity can be enhanced by a factor of $>1000$, as shown in Figure $4 \mathrm{~b}$. Together with its excellent tunability, the coupled system demonstrated in this work also provides an outstanding platform for surface-enhanced spectroscopy techniques.

Notably, when the gap size is large $(g>30 \mathrm{~nm})$, the near-field coupling becomes negligible and the coupling between the two plasmonic surfaces behaves like conventional thin-film interferences, except that there are additional phase shifts at the interfaces. This was confirmed by the simulated charge distributions shown in Figure 4. The charge distribution in the Au nanodisk array in the case of 

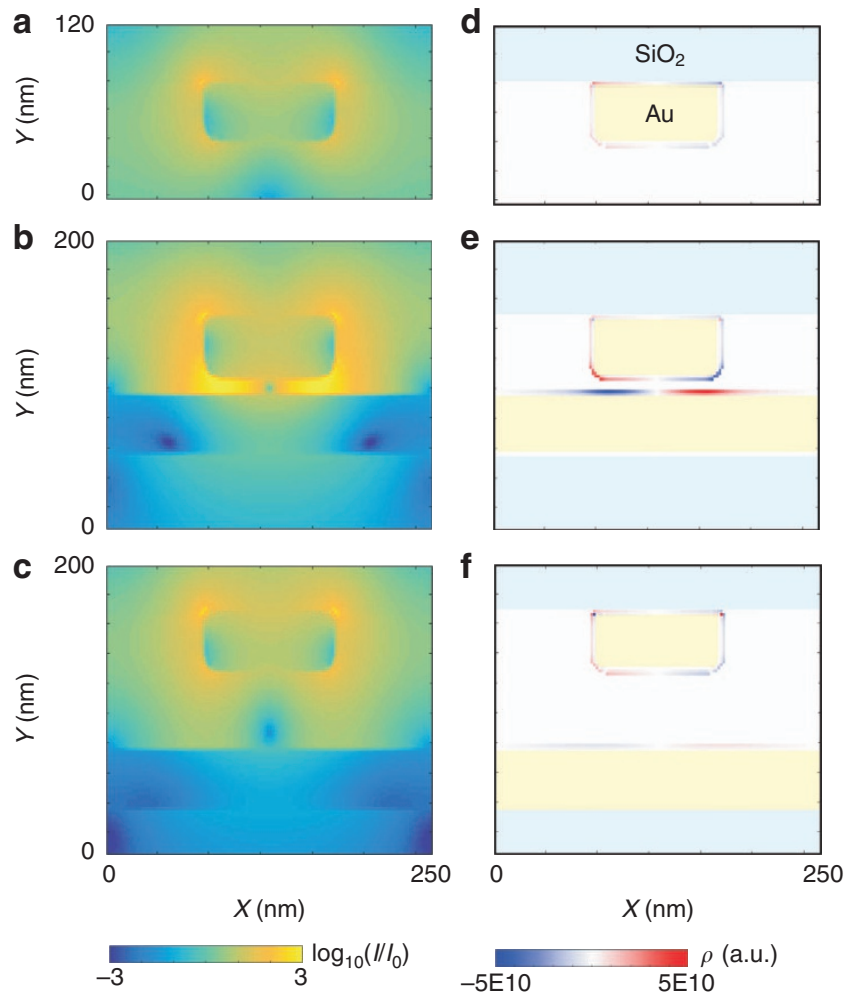

Figure 4 Simulated resonance behaviors of the coupled plasmonic metasurfaces. (a-c) show the field distributions in a unit cell of the coupled system with $g=\infty, 10$ and $50 \mathrm{~nm}$, respectively; (d-f) the respective charge distributions. In the simulation, a periodic boundary was used, with a 250$\mathrm{nm}$ pitch size; the unit Au nanodisk was $100-\mathrm{nm}$ wide and 40-nm thick; and the Au film was 40-nm thick.

$g=50 \mathrm{~nm}$ (Figure $4 \mathrm{f}$ ) is very similar to that in the case of $g=\infty$ (Figure $4 \mathrm{~d}$ ), without any of the charge concentration effect that is observed in the case of near-field coupling (Figure 4e). Interestingly, it has been reported that in the far-field coupling regime, the system can also exhibit good sensitivity to index change in the gap area ${ }^{43-45}$.

Finally, this technique is not limited to metal surfaces and, in principle, can also be used for inspecting dielectric surfaces because of the high sensitivity of plasmonic nanostructures to their local environment ${ }^{12}$. In particular, for surfaces of high refractive index, large resonance shifts and, consequently, high distance sensitivity can be expected ${ }^{46}$.

\section{CONCLUSIONS}

In this work, we studied the near-field coupling phenomena between 2D plasmonic nanostructures to develop a high-accuracy surface inspection technique. Using the method of Newton's rings, we were able to measure the dependence of the plasmon resonance modes on the gap size between a 2D Au nanodisk array and an Au thin film with nanometer precision. The results show that in the near-field regime $(g<30 \mathrm{~nm})$, the resonance frequency is extremely sensitive to the gap size. Even a slight change in the distance can alter the resonance wavelength significantly. When $g<10 \mathrm{~nm}$, the distance sensitivity $s=$ I $\mathrm{d} \lambda_{\text {res }} / \mathrm{d} g$ can reach $\sim 10$, which is 40 times higher than that in the farfield regime, where $s$ is only 0.23 . Moreover, analysis shows that $\lambda_{\text {res }}$ can be precisely determined using a numerical fitting technique, with an uncertainty as small as $0.03 \mathrm{~nm}$, corresponding to a $0.003 \mathrm{~nm}$ accuracy for distance measurements. With this high distance sensitivity and precision, we demonstrated a near-field couplingbased optical profiling technique that allows us to reveal nanometer-deep corrugations in a sample surface by simply placing that surface onto the developed Au nanodisk array-based plasmonic optical flat. This technique is fast, simple, accurate and capable of identifying nanometer corrugations; it is expected to find many applications in the fields of optical profiling and high-precision machining.

\section{CONFLICT OF INTEREST}

The authors declare no conflict of interest.

\section{AUTHOR CONTRIBUTIONS}

WZ conceived the idea and designed the experiments. JB, XW, JL and HG fabricated the samples. YZ performed all the measurements. PF and WZ performed the simulation. YZ, WZ and OM conducted the data analysis. WZ wrote the manuscript.

\section{ACKNOWLEDGEMENTS}

We gratefully acknowledge funding from the National Natural Science Foundation of China (Nos. 11374152, 11574142 and 11321063), the National Key Technologies R\&D Program of China (No. 2016YFA0201104), the National Basic Research Program of China (No. 2015CB659400) and the Priority Academic Program Development (PAPD) of Jiangsu Higher Education Institutions.

1 Yoshizawa T. Handbook of Optical Metrology: Principles and Applications. 2nd edn. Boca Raton: CRC Press; 2015.

2 Bewoor AK, Kulkarni VA. Metrology \& Measurement. New Delhi: Tata McGraw-Hill Education Pvt. Ltd.; 2009.

3 Newton I. Opticks. London: Sam. Smith and Benj. Walford; 1704. pp 110-112.

4 Malacara D. Optical Shop Testing. 3rd ed. Hoboken: A John Wiley \& Sons, Inc.; 2007.

5 Novotny L, Hecht B. Principles of Nano-Optics. New York: Cambridge University Press; 2006

6 Pohl DW, Denk W, Lanz M. Optical stethoscopy: image recording with resolution $\lambda 20$. Appl Phys Lett 1984; 44: 651-653.

7 Lewis A, Isaacson M, Harootunian A, Muray A. Development of a 500- $\AA$ spatialresolution light-microscope: I. Light is efficiently transmitted through $\lambda / 16$ diameter apertures. Ultramicroscopy 1984; 13: 227-231.

8 Fang $\mathrm{N}$, Lee $\mathrm{H}$, Sun $\mathrm{C}$, Zhang $\mathrm{X}$. Sub-diffraction-limited optical imaging with a silver superlens. Science 2005; 308: 534-537.

9 Maier SA. Plasmonics: Fundamentals and Applications. New York: Springer; 2007

10 Schuller JA, Barnard ES, Cai WS, Jun YC, White JS et al. Plasmonics for extreme light concentration and manipulation. Nat Mater 2010; 9: 193-204.

11 Lal S, Link S, Halas NJ. Nano-optics from sensing to waveguiding. Nat Photonics 2007 ; 1: 641-648.

12 Willets KA, Van Duyne RP. Localized surface plasmon resonance spectroscopy and sensing. Annu Rev Phys Chem 2007; 58: 267-297.

13 Prodan E, Radloff C, Halas NJ, Nordlander P. A hybridization model for the plasmon response of complex nanostructures. Science 2003; 302: 419-422.

14 Nordlander P, Oubre C, Prodan E, Li K, Stockman MI. Plasmon hybridizaton in nanoparticle dimers. Nano Lett 2004; 4: 899-903.

15 Lal S, Grady NK, Goodrich GP, Halas NJ. Profiling the near field of a plasmonic nanoparticle with Raman-based molecular rulers. Nano Lett 2006; 6: 2338-2343.

16 Jain PK, Huang W, El-Sayed MA. On the universal scaling behavior of the distance decay of plasmon coupling in metal nanoparticle pairs: a plasmon ruler equation. Nano Lett 2007; 7: 2080-2088.

17 Tabor C, Murali R, Mahmoud M, El-Sayed MA. On the use of plasmonic nanoparticle pairs as a plasmon ruler: the dependence of the near-field dipole plasmon coupling on nanoparticle size and shape. J Phys Chem A 2009; 113: 1946-1953.

18 Jain PK, El-Sayed MA. Surface plasmon coupling and its universal size scaling in metal nanostructures of complex geometry: elongated particle pairs and nanosphere trimers. J Phys Chem C 2008; 112: 4954-4960.

19 Tabor C, Van Haute D, El-Sayed MA. Effect of orientation on plasmonic coupling between gold nanorods. ACS Nano 2009; 3: 3670-3678.

20 Liu N, Hentschel M, Weiss T, Alivisatos AP, Giessen H. Three-dimensional plasmon rulers. Science 2011; 332: 1407-1410.

21 Shafiei F, Wu C, Wu YW, Khanikaev AB, Putzke $P$ et al. Plasmonic nano-protractor based on polarization spectro-tomography. Nat Photonics 2013; 7: 367-372. 
22 Hill RT, Mock JJ, Hucknall A, Wolter SD, Jokerst NM et al. Plasmon ruler with angstrom length resolution. ACS Nano 2012; 6: 9237-9246.

23 Hill RT, Kozek KM, Hucknall A, Smith DR, Chilkoti A. Nanoparticle-film plasmon ruler interrogated with transmission visible spectroscopy. ACS Photonics 2014; 1 : 974-984.

24 Moreau A, Ciracì C, Mock JJ, Hill RT, Wang Q et al. Controlled-reflectance surfaces with film-coupled colloidal nanoantennas. Nature 2012; 492: 86-89.

25 Schmidt MA, Lei DY, Wondraczek L, Nazabal V, Maier SA. Hybrid nanoparticlemicrocavity-based plasmonic nanosensors with improved detection resolution and extended remote-sensing ability. Nat Commun 2012; 3: 1108.

26 Lei DY, Fernández-Domínguez AI, Sonnefraud Y, Appavoo K, Haglund RF et al. Revealing plasmonic gap modes in particle-on-film systems using dark-field spectroscopy. ACS Nano 2012; 6: 1380-1386.

27 Kats MA, Blanchard R, Genevet P, Capasso F. Nanometre optical coatings based on strong interference effects in highly absorbing media. Nat Mater 2013; 12: 20-24.

$28 \mathrm{Yu}$ NF, Capasso F. Flat optics with designer metasurfaces. Nat Mater 2014; 13: 139-150.

29 Song HM, Guo LQ, Liu ZJ, Liu K, Zeng X et al. Nanocavity enhancement for ultra-thin film optical absorber. Adv Mater 2014; 26: 2737-2743.

30 Tsai DP, Lu YY. Tapping-mode tuning fork force sensing for near-field scanning optical microscopy. Appl Phys Lett 1998; 73: 2724-2726.

31 Karrai K, Grober RD. Piezoelectric tip-sample distance control for near-field optical microscopes. Appl Phys Lett 1995; 66: 1842-1844.

32 Sönnichsen C, Geier S, Hecker NE, von Plessen G, Feldmann J et al. Spectroscopy of single metallic nanoparticles using total internal reflection microscopy. Appl Phys Lett 2000; 77: 2949-2951.

33 Kottmann JP, Martin OJF, Smith DR, Schultz S. Field polarization and polarization charge distributions in plasmon resonant nanoparticles. New J Phys 2000; 2: 27.

34 Ameling R, Giessen H. Microcavity plasmonics: strong coupling of photonic cavities and plasmons. Laser Photonics Rev 2013; 7: 141-169.

35 Lévêque G. Martin OJF. Optical interactions in a plasmonic particle coupled to a metallic film. Opt Express 2006; 14: 9971-9981.

36 Ciracì C, Hill RT, Mock JJ, Urzhumov Y, Fernández-Domínguez Al et al. Probing the ultimate limits of plasmonic enhancement. Science 2012; 337: 1072-1074.
37 Bevington PR, Robinson DK. Data Reduction and Error Analysis for the Physical Sciences. 3rd edn. New York: McGraw-Hill; 1994.

38 Rust MJ, Bates M, Zhuang XW. Sub-diffraction-limit imaging by stochastic optical reconstruction microscopy (STORM). Nat Methods 2006; 3: 793-795.

39 Betzig E, Patterson GH, Sougrat R, Lindwasser OW, Olenych S et al. Imaging intracellular fluorescent proteins at nanometer resolution. Science 2006; 313: $1642-1645$

40 Xu XY, Kedem Y, Sun K, Vaidman L, Li CF et al. Phase estimation with weak measurement using a white light source. Phys Rev Lett 2013; 111: 033604.

41 Chou SY, Krauss PR, Renstrom PJ. Imprint lithography with 25-nanometer resolution. Science 1996; 272: 85-87.

42 Li ZW, Gu YN, Wang L, Ge HX, Wu W et al. Hybrid nanoimprint-soft lithography with sub-15 nm resolution. Nano Lett 2009; 9: 2306-2310.

43 Ameling R, Langguth L, Hentschel M, Mesch M, Braun PV et al. Cavity-enhanced localized plasmon resonance sensing. Appl Phys Lett 2010; 97: 253116.

44 Artar A, Yanik AA, Altug H. Fabry-peacuterot nanocavities in multilayered plasmonic crystals for enhanced biosensing. App/ Phys Lett 2009; 95: 051105.

45 Chanda D, Shigeta K, Truong T, Lui E, Mihi A et al. Coupling of plasmonic and optical cavity modes in quasi-three-dimensional plasmonic crystals. Nat Commun 2011; 2: 479.

46 Zhang WH, Martin OJF. A universal law for plasmon resonance shift in biosensing. ACS Photonics 2015; 2: 144-150.

cc)(i) (2) This work is licensed under a Creative Commons Attributionc. ${ }_{\mathrm{BY}} \mathrm{NC}$ SA NonCommercial-ShareAlike 4.0 International License. The images or other third party material in this article are included in the article's Creative Commons license, unless indicated otherwise in the credit line; if the material is not included under the Creative Commons license, users will need to obtain permission from the license holder to reproduce the material. To view a copy of this license, visit http://

creativecommons.org/licenses/by-nc-sa/4.0/

(C) The Author(s) 2016

Supplementary Information for this article can be found on the Light: Science \& Applications' website (http://www.nature.com/lsa). 